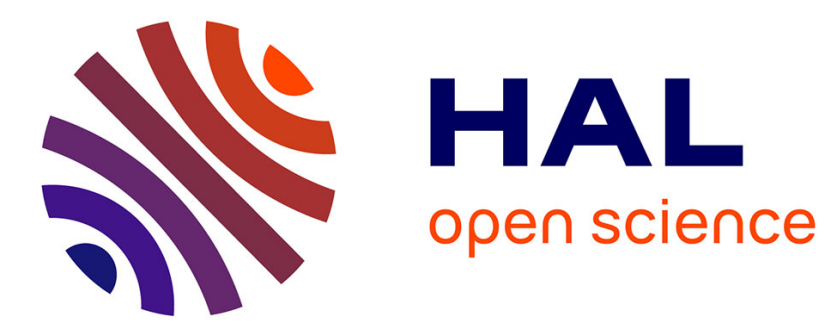

\title{
Un nouveau monument funéraire gallo-grec à Beaucaire (Ugernum)
}

Jean-Claude Bessac, Yves Gasco, André Michelozzi, Michel Lejeune

\section{To cite this version:}

Jean-Claude Bessac, Yves Gasco, André Michelozzi, Michel Lejeune. Un nouveau monument funéraire gallo-grec à Beaucaire (Ugernum). Gallia - Fouilles et monuments archéologiques en France métropolitaine, 1986, 44 (1), pp.55-64. 10.3406/galia.1986.2852 . hal-01940885

\section{HAL Id: hal-01940885 \\ https://hal.science/hal-01940885}

Submitted on 27 Feb 2020

HAL is a multi-disciplinary open access archive for the deposit and dissemination of scientific research documents, whether they are published or not. The documents may come from teaching and research institutions in France or abroad, or from public or private research centers.
L'archive ouverte pluridisciplinaire HAL, est destinée au dépôt et à la diffusion de documents scientifiques de niveau recherche, publiés ou non, émanant des établissements d'enseignement et de recherche français ou étrangers, des laboratoires publics ou privés.

\section{(ㅇ)(1) $\$$}

Distributed under a Creative Commons Attribution - NonCommercial - NoDerivatives| 4.0 


\title{
UN NOUVEAU MONUMENT FUNÉRAIRE GALLO-GREC À BEAUCAIRE (Ugernum)
}

\author{
par Jean-Claude BESSAC, Yves GASCO, André MICHELOZZI \\ et par Michel LEJEUNE
}

Trouvée fortuitement il y a quelques années par un paysan au cours d'un labour, repérée et étudiée en 1984 par des spécialistes beaucairois, mais demeurée propriété de la famille de l'inventeur, la partie supérieure inscrite d'un monument funéraire d'époque gallo-grecque vient, tant aux archéologues qu'aux linguistes, apporter du nouveau et poser des questions.

Le chapiteau est ci-après publié conjointement par J.-C. Bessac (CNRS), Y. Gasco (conservateur du musée municipal contrôlé de Beaucaire), A. Michelozzi (correspondant de la Direction des Antiquités du Languedoc-Roussillon); l'épitaphe par Michel Lejeune.

\section{LE MONUMENT}

\section{LOCALISATION}

Un repérage systématique des sites gallo-romains sur la commune de Beaucaire (Gard), à l'occasion d'une étude plus vaste a permis la découverte d'un chapiteau à inscription gallo-grecque. Nous devons à l'amabilité de la propriétaire d'avoir pu l'étudier et tenter une opération de moulage ${ }^{2}$.

Le gisement, situé auprès du Mas Peirette (fig. 1) dans le quartier dit Mas de Maillan ${ }^{3}$, présente des vestiges antiques abondants (Chalcolithique, Gallo-romain du $\mathrm{i}^{\mathrm{er}}$ s. av. J.-C. au ve s. ap. J.-G. $)^{4}$ épandus sur trois hectares, avec une plus grande densité sur un hectare (fig. 2). Il est comparable à bien d'autres sites découverts ou redécouverts lors de nos prospections.

1 J.-C. Bessac, M. Christol, J.-L. Fiches, Y. Gasco, M. Janon, A. Micheiozzi, C. Raynalin, A. Roth-Conges et D. Terrer, Etudes sur Ugernum. Beaucaire el le beaucairois à l'époque romaine, à paraître. ici.

$2 \mathrm{M}^{\mathrm{me}} \mathrm{Vve}$ Cadène, Beaucaire, que nous tenons à remercier

3 Coordonnées Lambert : feuille Nimes, XXIX 42, 7/8, zone III, 782, 125/168, 500 el $782,350 / 168,575$; altitude : 3 à $5 \mathrm{~m}$. - Cadastre : Beaucaire, 1940/1983, section $H$ dite des carrières, feuille 2, lieu-dit Mas de Maillan, parcelles 637 et 638 principalement.

4 Mobilier déposé au Musée municipal contrôlé de La Vignasse, Beaucaire (Gard) sous les nos 979-1-4753 à 4773 pour les anciennes trouvailles; au dépôt archéologique, 76 , rue de Nîmes pour les nouvelles prospections et moulage du chapileau déposé au Musée municipal contrôlé de La Vignasse sous le no $986-1$. 
1 Le site d'Ugernum. Hachures verticales : habitat urbain du ${ }^{\text {er }} \mathbf{S}$. av. J.-C. ; hachures obliques : zone des nécropoles ; E : Ecluse ; B : Brasserie; '': Mas Peirette; J : Mas de Jallon. (Carte IGN : 1/50.000e).

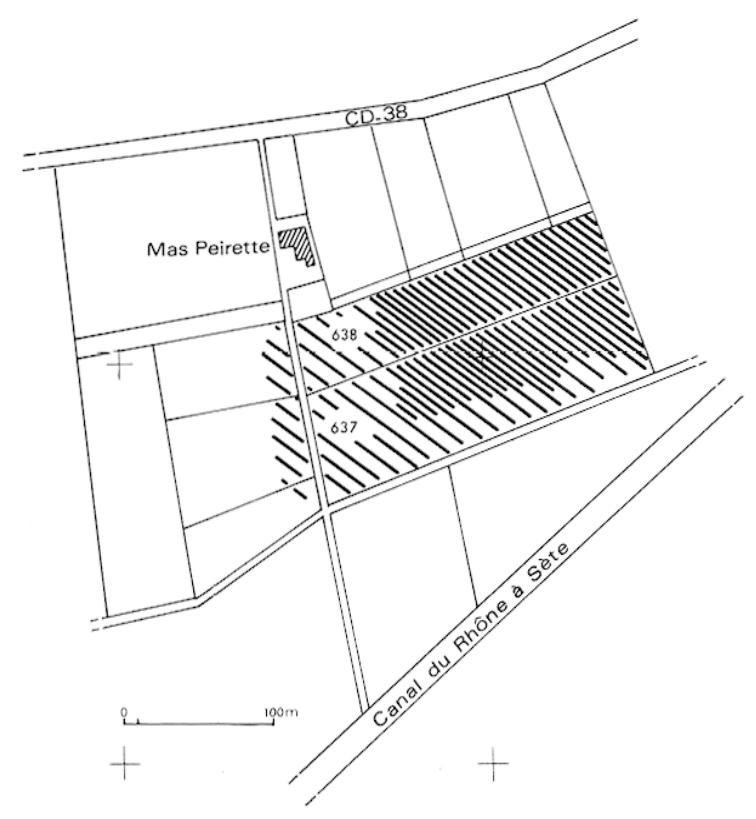

2 Le Mas Peirette. Extrait du plan cadastral, Beaucaire (Gard) 1940/1983, $\leftarrow$ section $\mathrm{H}$. 
Il est situé en plaine, au contact du talus qui termine le plateau des Costières sur la vallée du Rhône, à l'apparition des alluvions fines, caractéristiques de sa très faible altitude $(3 \mathrm{à} 5 \mathrm{~m})$. Il reste proche de la ville antique puisque, vers le s.-o., le Mas Peirette (comme vers l'o., le Mas de Jallon ${ }^{5}$, site d'une tombe du $\mathrm{I}^{\mathrm{er}} \mathrm{s}$. av. J.-C.) n'est distant d'Ugernum que de $3 \mathrm{~km}$ environ. Il est plus proche encore $(2 \mathrm{~km})$ de la zone des nécropoles ${ }^{6}$ : Sizen, Colombes, Marronniers. De plus, la colonne monolithique dite de la Brasserie aurait été vraisemblablement découverte in silu ${ }^{7}$, au pied des Costières, près d'une source vauclusienne, à 1,200 km du Mas Peirelle.

\section{DESCRIPTION}

Le chapileau est taillé dans un calcaire coquillier tendre, blanchâtre, très vraisemblablement d'origine locale, que l'on nomme "pierre de Beaucaire ". Il présente des stries de tournage très nettes sur toute sa partie cylindrique. La partie supérieure, de forme conique, est creusée de cannelures taillées transversalement au ciseau de façon assez irrégulière.

A mi-hauteur de la partie cannelée de ce cône, les génératrices détcrminent unc légère dépression d'un demi-centimètre : effet volontaire pour amorcer le redressement de la partie supérieure ou erreur au cours de la taille, qui est du reste assez négligée ? Cette dernière hypothèse n'est pas impossible lorsque l'on voit l'hésitation qui a présidé à l'établissement des dimensions propres ou relatives des cannelures.

Les lits d'attente (?) et de pose sont très abîmés, ce dernier plus fortement en raison des travaux agricoles qui ont mené à sa découverte. Il subsiste en leur centre une cavité quadrangulaire d'environ $9 \mathrm{~cm}$ sur $10 \mathrm{~cm}$, profonde de $6 \mathrm{~cm}$ à la base et de $9 \mathrm{~cm}$ au sommet. Il pourrait s'agir de l'ancrage carré du tour qui a servi à façonner la partie cylindrique. Ces deux cavités étaient comblées par du mortier moderne, qu'il s'agisse du socle de béton pour la partie sommitale ou d'un galet décoratif pour l'autre extrémité, le chapiteau étant conservé présenté à l'envers (fig. 3,4 et 5).

La modénalure est assez difficile à établir de façon rigoureuse tant en raison de l'originalité de l'ensemble que de l'imprécision de la taille.

Du lit de pose se développe une partie cylindrique, fût de colonne ou gorgerin, il est impossible de trancher. En effet les deux solutions sont attestées (cf. infra), l'astragale étant même connue sans gorgerin sur la pièce de la Brasserie de Beaucaire.

Un petit congé dégage un bandeau en léger ressaut, puis une échine en demi-cœur mal séparée de l'élément précédent en raison de la médiocrité du tournage. L'échine elle-même est composée sur la presque totalité de son tracé de deux éléments séparés par un filet. S'agit-il d'un défaut de tournage ou de deux échines en demi-cœur superposées ? De plus, sur quelques centimètres, le bandeau et la première échine sont indifférenciés, formant un élément de type scotie droite ou plutôt cavet, en un relief masquant le raccord cylindre/échine. La forme hésiterait donc entre celles, attestées comme

5 P. GакмY, A. Michriozzi el M. P'Y, Une nouvelle sépulture protohistorique $\dot{a}$ Beaucaire (Gard): la tombe du mas de Jallon dans R.A.N., 1981, XIV, p. 71-87, 9 fig.

6 j. Juliax el M. Lovis, Catalogue des mobiliers funéraires des sépullures antiques du Musée de Beaucaire dans Cahiers d'Histoire et d'Archéologie, 1934, p. 193-227, 6 fig. (zone dite "du Sizen"). - B. Denet, A. Mrchelozzi et M. $\mathrm{PY}$, La nécropole des Colombes à Beaucaire (Gard) $\left(\mathrm{I}^{\mathrm{e}}\right.$ $I^{\text {er }}$ s. av. J.-C.) dans R.A.N., 1974, 1. VII, p. 59-118, 52 fig., annexe de H. Huday. - B. Dedet, A. Mrchelozzi, M. Py, C. Raynaud et C. Tendille, Ugernum, protohistoire de Beaucaire dans Cahier de l'A.R.A.L.O. 1978, no 6, 156 p., 87 fig., annexe de M. LEJEUnE.

7 L'emplacement pourrait correspondre à l'ancien SaintSixte, ancien prieuré aujourd'hui disparu, où Vincent Sève a signalé une tombe gallo-romaine au xvile s. (A. Eyssetre, Histoire administrative de Beaucaire, tome I. - Beaucaire, 1884, 476 p. ; voir p. 444-446). 


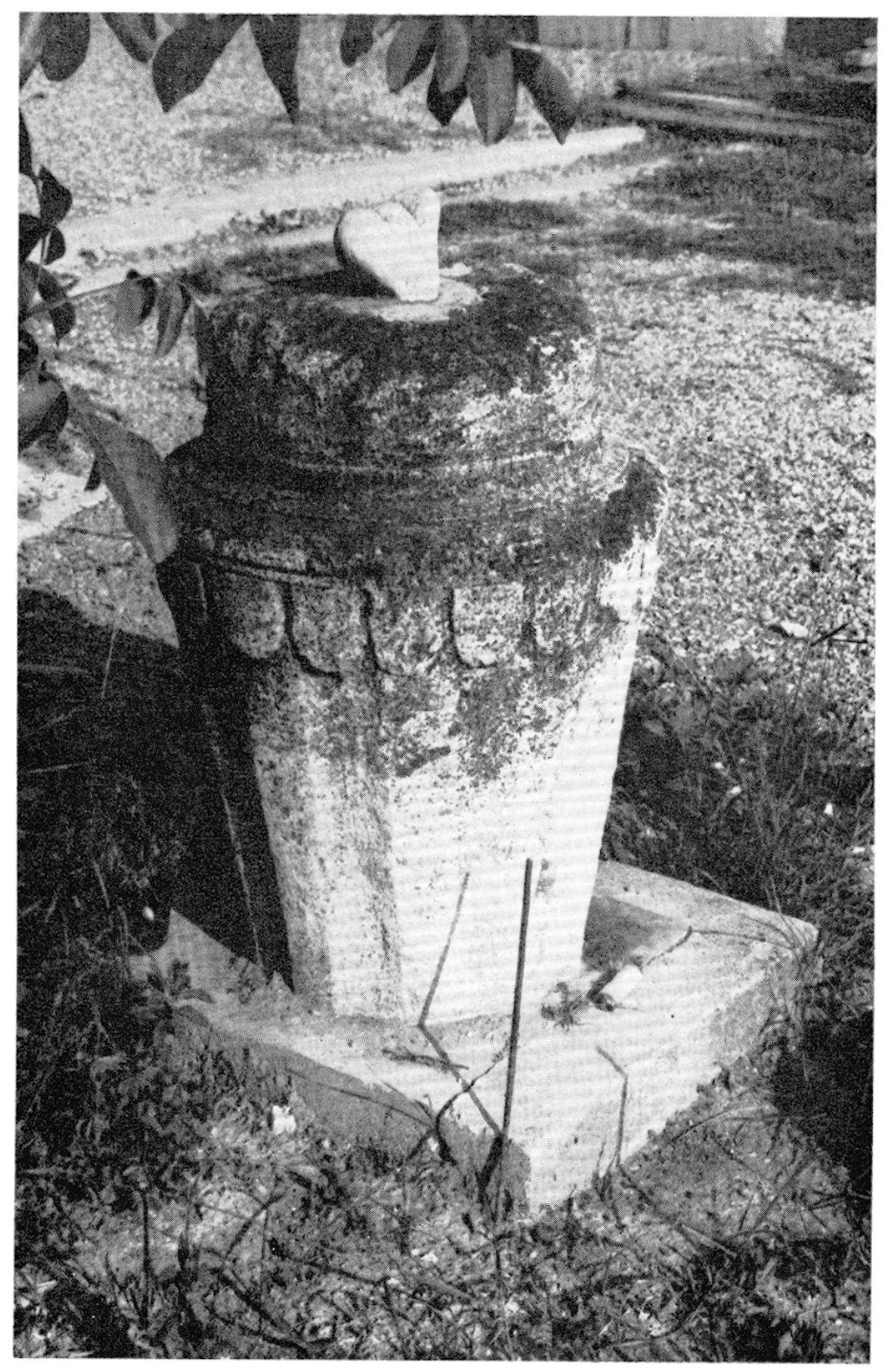

3 Le Mas Peirelte. Le monument photographié lors de sa découverte.

support d'inscriptions gallo-grecques, d'inspiration dorique et celles plus proches du type toscan (échine en quart-de-rond) qui représentent peut-être une étape de la tendance qui a conduit aux chapiteaux de type toscan provincial, type DC de P. Broise ${ }^{8}$, avec échine en doucine, bien connus au milieu du $\mathrm{I}^{\text {er }}$ s. ap. J.-C. sur la même aire géographique ${ }^{9}$.

8 P. Brorsf, Élements d'un ordre toscan provincial en Haule-Savoie, dans Gallia, 27, 1969, p. 15-22; voir p. 17, fig. 1 .

9 Chapiteau du mas des Tourelles, Beaucaire, à paraitre dans Eludes sur Gigernum, op. cit. - Chapiteaux 1, 7 et 8 de la maison au Dauphin, ef. Chr. Goudreat, Les fouilles de la Maison au Dauphin. Recherches sur la romanisalion de Vaison-la-Romaine, 37c Supplément à Gallia, 1979. 


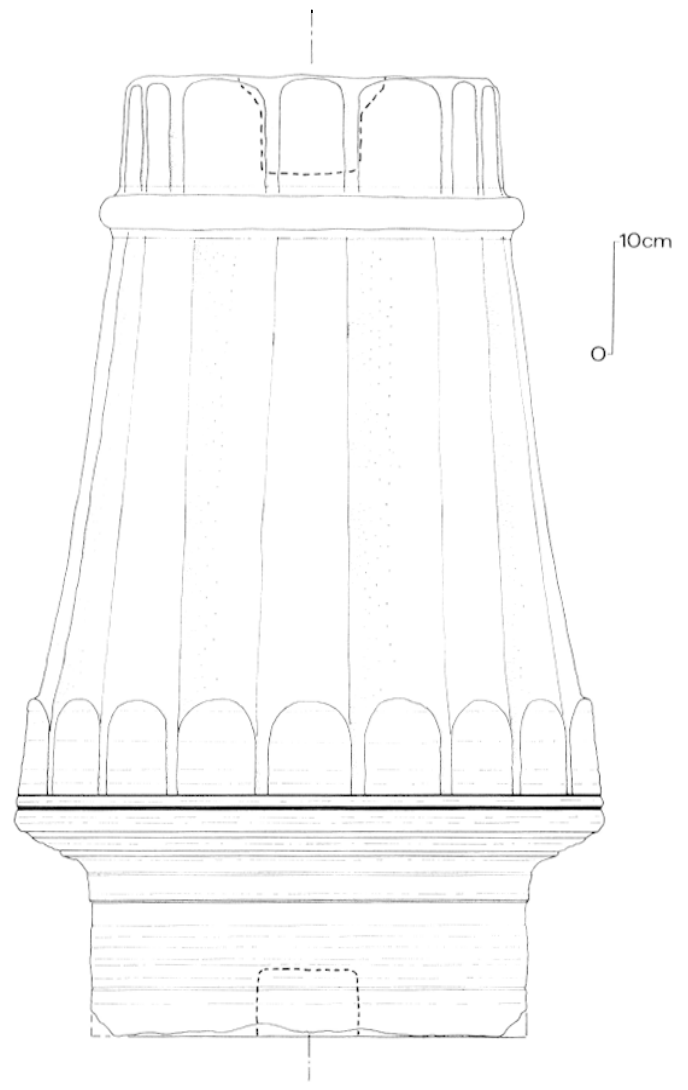

4 Relevé graphique du bloc; en épais tireté, les cavités creusées aux extrémités.

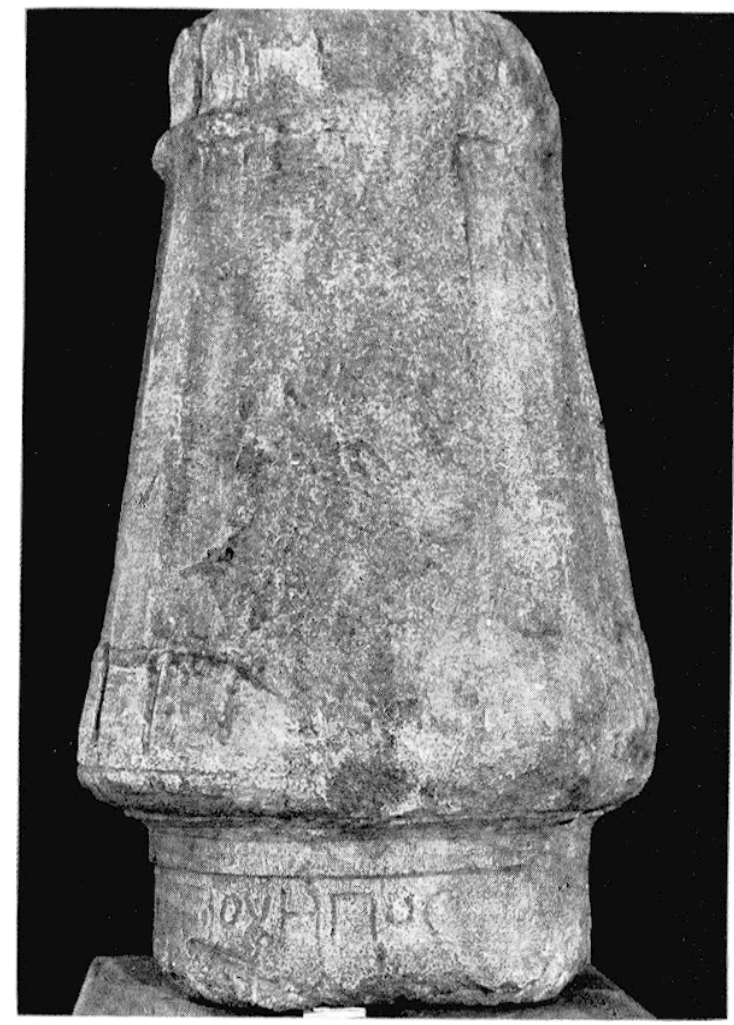

5 Photographie du moulage du monument.

Cette partie d'allure dorique se raccorde au trone de cône cannelé par un filet amorçant un tore peu individualisé, lui-même terminé et suivi par deux rainures.

Le tronc de cône se poursuit par un décor en U pleins renversé formant un léger relief d'où jaillissent de larges cannelures irrégulières et peu profondes. Leurs dimensions n'étant pas équivalentes, il y a un net décalage entre ces deux éléments.

Le bloc se termine au sommet par la répétition du motif en Li renversés, séparé des cannelures par un tore amené de chaque côté par un filet mal venu, pcut-être dû simplement à la négligence de lia taille.

\section{Comparaisons}

Tel quel, ce bloc suggère un chapiteau papyriforme, impression renforcée par une hypothèse de reconstitution du monument mettant l'inscription à hauteur d'yeux et portant l'ensemble à $2,5 \mathrm{~m}$ de haut. Le monument prendrait alors une allure élancée (fig. 6) bien différente de la colonne de la Brasserie de Beaucaire. Il existe il est vrai des inscriptions situées nettement plus bas ; cependant, dans ce cas, le surplomb de l'échine ne favoriserait guère la lecture.

En fait, découvert dans un contexte gallo-grec, il peut être décomposé en trois éléments ; une colonne; un chapiteau d'inspiration dorique dont l'abaque, absent, serait peut-être remplacé par un tore peu dégagé; enfin un tronc de cône cannelé décoré au sommet et à la base de festons. Il n'est pas 
nécessaire de voir un autre élément au sommet, les festons terminant convenablement l'ensemble ${ }^{10}$, et le tore n'étant pas forcément l'astragale d'une partie absente.

Si les deux premiers éléments du bloc, ainsi que la technique de taille et l'emplacement de l'inscription trouvent des comparaisons, le troisième élément semble encore inconnu dans notre région.

- A Beaucaire ont été mis au jour trois chapiteaux à inscription gallo-grecque. Deux en juillet 1809 , "dans les fouilles de l'emplacement de l'éperon de mouille de l'écluse de prise d'eau du Rhône du canal de Beaucaire à Aiguesmortes "11, monuments disparus depuis lors, et un troisième remarqué en 1962 dans les bâtiments administratifs de la Brasserie de La Meuse ${ }^{12}$.

Seul ce dernier subsiste, partie intégrante d'une colonne monolithe conservée au musée municipal ${ }^{13}$. Cette colonne, d'une technique aussi négligée faisant appel au tour, présente une modénature semblable : un tore mal dégagé (astragale ?) y remplace la bande terminant le fût ; l'échine est d'une seule venue.

Quant aux deux précédents chapiteaux, les représentations que nous en avons restent assez contradictoires. Seule certitude : leur appartenance à cette série dorico-toscane provinciale d'époque républicaine.

- Région: le traitcment du raccord fût-chapitcau de l'exemplaire du Mas Peirette, malgré ses hésitations, n'est pas sans rappeler celui des monuments gallo-grecs de Saint-Gilles (Gard) ${ }^{14}$ peu éloigné géographiquement, ou de Subslantion à Gastelnau-le-Lèz (Hérault) ${ }^{15}$ sur la route d'Espagne. Ces derniers, de style plutôt toscan, utilisent un cavet.

Des quelque sept chapiteaux à inscription gallo-grecque connus surmontant des colonnes ${ }^{16}$, trois ont disparu, mais tous provenaient du Bas-Languedoc. Toutefois, sur trois colonnes connues ${ }^{17}$, deux proviennent de Provence. Quant aux chapiteaux de piliers ou pilastres, on peut les réduire à trois, tous languedociens ${ }^{18}$, le pilier mouluré de Malaucène (Vaucluse) ${ }^{19}$ se rapprochant assez par sa taille, $1,18 \mathrm{~m}$, et sa forme, d'un autel funéraire. Ces autels à inscription gallo-grecque ne sont pour le moment connus qu'en Provence, au nord des Alpilles ${ }^{20}$ (fig. 7).

10 Dimensions : diamètre fût $=425 \mathrm{~mm}$; diamètre maximal $=575 \mathrm{~mm} ;$ diamètre partie supérieure $=360 \mathrm{~mm}$; hauteur totale $=855 \mathrm{~mm}$.

$11 \mathrm{~J}$. J. Trelis, Notice des travaux de l'Academie du Gard pendant l'année 1809, Nîmes, 1810, p. 410. - C. Blaun, Antiquités de la Ville de Beaucaire, Alais, 1819, p. 26, pl. VII. - A. Eyssette, op. cit. - P. Eyssette, Notice sur une inscription découverte à Beaucaire vers 1809 et sur les vestiges d'un monument funéraire, 35c Congrès scientifique de France, Montpellier, 1872, II, p. 383. - E. Germer-Durand, D'une prétendue inscription grecque de Beaucaire dans Mémoires de l'Académie du Gard, 1866-1867, Nîmes, 1868, p. 249-263. - P.-M. Duval, Beaucaire et les inscriptions gallo-grecques dans le département du Gard. - Bulletin de la Société d'Hisloire et d'Archéologie de Beaucaire, 7, 1962, p. 16-17. - Id., line nouvelle inscription gallo-grecque dans le département du Gard dans Bulletin de la Société Nationale des Antiquaires de France, 1962, p. 114-116. - O. Lombard et J. Roche, Les inscriplions cello-grecques de Beaucaire dans Bulletin de la Sociélé d'IIistoire et d'Archéologie de Beaucaire, 17, 1966. - M. Lejeune, Les chapiteaux de Beaucaire $\grave{a}$ inscriptions gallo-grecques dans dans B. Dedel, A. Michelozzi..., 1978, op. cil., p. 139-156. - M. LeJeune, Recueil des inscriptions gauloises (R.1.G.), I, texles gallo-grecs. $X L V^{\mathrm{c}}$ supplement à Gallia, 1985, p. 223228.

12 P.-M. Duval, op. cit., 1962. - Id. dans Gallia, XXII, 2, 1964, p. 438-499. - o. Lombard et J. Roche, op. cit.,
1966. - M. Lejeune, op. cit., 1978. - Id., op. cit., 1985.

13 No Inventaire : 979-1-407.

14 Gallia, XX, Informations, 1962, p. 636. - Ibid., XXII, 1964, p. 505. - Ibid., XXIX, 1971, p. 400. - Ibid., XXXI, 1973, p. 511. - Ibid., XXXVI, 1978, p. 457.

15 A. Héron de Villefosse, Bulletin archéologique du comité des travaux historiques, Paris, 1887, p. 202. - E. BonNeT, Antiquilés el monuments ... de l'Hérault, 1905, p. 22. J.-C. RichaRd, La région mont pelliéraine à l'époque préromaine, 750-121 av. J.-C., dans Coll. Latomus, vol. 130, Bruxelles, 1973,164 p., 18 fig.

16 Gard : trois à Beaucaire, dont deux perdus, cf. M. LeJeune, R.I.G., 1, G. 161 à 163 ; un à Nîmes (perdu), ibid., G. 205 ; un à Saint-Gilles, ibid., G. 216 ; Hérault : un à Castelnau-le-Lèz, ibid., G. 221; un à Montagnac, ibid, G 224.

17 Gard : un fragment à Sernhac, ibid., G. 217; Vaucluse : un fragment à Apt, ibid., G. 110 ; une colonne à L'Isle-surSorgues, ibid., G. 147.

18 Gard : un à Nîmes, ayant servi de socle de statue, ibid., G. 203 ; un à Saint-Côme-et-Maruéjols, ibid., G. 214; un à Lzès, ibid., G. 219. L'exemplaire d'Alise-Saint-Reine, ibid., G. 256, très éloigné géographiquement au moins peul être considéré à part.

19 Malaucène, Vaucluse, ibid., G. 148.

20 Bouches-du-Rhône : trois à Glanum, Saint-Rémy-deProvence, ibid., G. 64,65 et 67 ; un à Orgon, ibid., G. 27. 


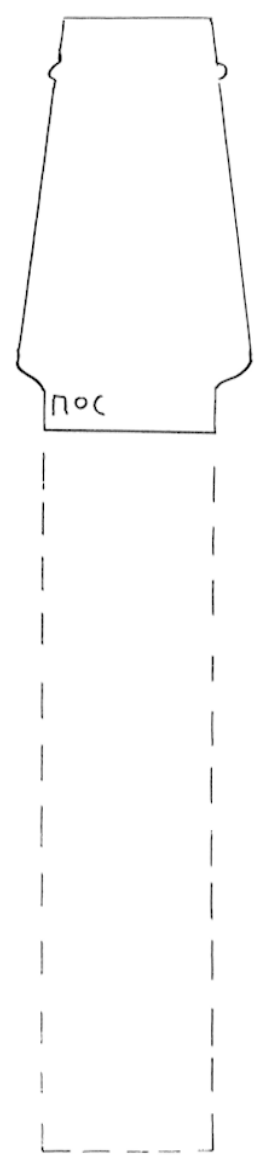

6 Essai de restitution du profil du monument (en lui supposant $250 \mathrm{~cm}$ de hauteur et en faisanl abstraction de la forme, non connue, de la base).

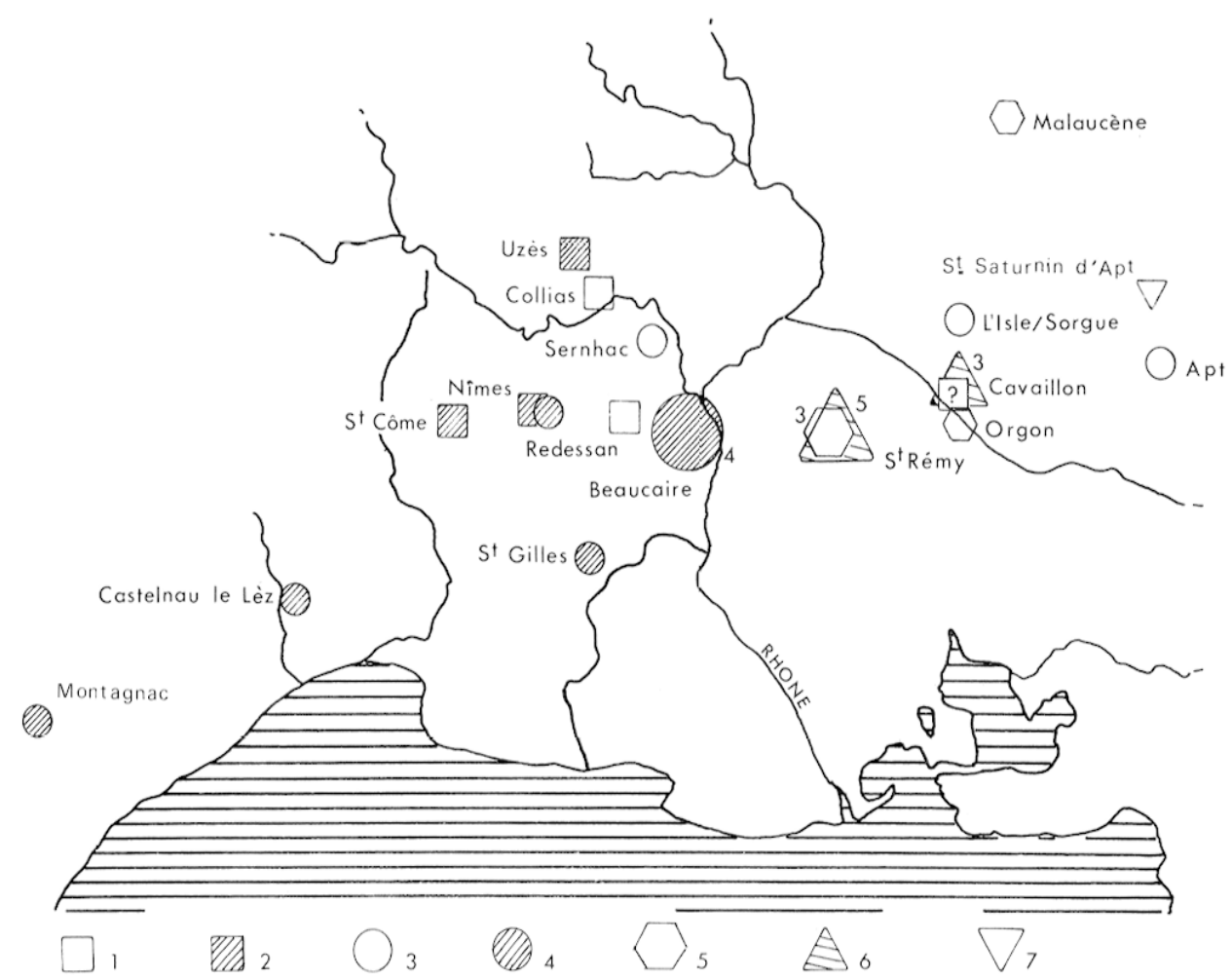

7 Répartition de quelques monuments épigraphes. 1 : piliers ; 2 : piliers à chapiteaux ; $3:$ colonnes ; 4 : colonnes à chapiteaux ; 5 : autels; 6 : cippes à fronton; 7 : cippe de Saint-Saturnin d'Apt. 
La partie inférieure du bloc découvert au Mas Peirette s'intègre donc dans cette série. Ce n'est pas le cas de la partie supérieure. Seule sa forme générale peut évoquer des monuments connus, mais nous ne connaissons pas de comparaison directe.

On peut donc rappeler deux tendances, une d'origine celtique et ses vraisemblables avatars provençaux, l'autre d'origine hellénique. Les «stèles » coniques ou pyramidales existent dans le milieu celtique où elles auraient peul-être même duré jusqu'à La Tène finale. Une série importante existe en Armorique ${ }^{21}$. On ne peul éviler un rapprochement avec les piliers à fronton, épigraphes ou non, si largement représentés au nord des $\Lambda$ lpilles, qui s'effilent vers le sommet ${ }^{22}$.

L'aulre tendance est celle des stèles ou colonnes funćraires plus ou moins phalliques, tronconiques et lerminées par acanthes ou pommes de pin bien connues aussi dans le monde étrusque ${ }^{23}$. Un cippe de Saint-Salurnin d'Apt (Vaucluse) entrerait assez bien dans cette série ${ }^{24}$.

On ne peut terminer ce rapide survol sans évoquer le chapiteau de Montagnac (Héraull) ${ }^{25}$. Élément lourné, il présente une échine d'allure dorique décorée de gravures en chevron suggérant des oves (?) mais l'abaque est absente, remplacée par des bandeaux décorés surmontés d'un tronc de còne aplati. L'inscription court sous l'astragale, soit au sommet de la colonne.

\section{L'INSCRIPTION}

Elle est composée d'une scule série de huit lettres (au moins) tracées puis incisées à l'aide d'un gravelet guidé par une main experte. Il est probable que l'ouvrier qui a taillé le chapiteau n'est pas celui qui a soigné ces lettres (fig. 8).

Cet ensemble se situe dans la partie haute du tambour. Il n'y a pas trace d'une seconde ligne sur la partie du monument conservée. On remarque habituellement l'utilisation comme champ épigraphique de l'abaque : sur onze chapiteaux, neuf fois plus une douteuse (Uzès : les moulures sont-elles au-dessus de l'abaque ou représentent-clles le chapitcau, l'inscription étant alors sur le pilier ?). Par contre, il est vrai que le tronc de cône ne fournissait pas un bon support, comme c'est le cas pour l'exemplaire de Montagnac, inscrit sous l'astragale. Cela se retrouve aussi sur colonnes ou fragments de colonnes.

$$
\text { J.-C. B.; Y. G.; A. M. }
$$

\section{L'ÉPITAPHE*}

L'inscription court horizontalement sur le tambour (haut de $110 \mathrm{~mm}$ ) solidaire du chapiteau, et occupe une longueur de $350 \mathrm{~mm}$, soit le quart de la circonférence. Lettres de 48 à $52 \mathrm{~mm}$ (sauf les o, petits : 28 à $30 \mathrm{~mm}$ ). Hauts des lettres à $20 \mathrm{~mm}$ environ sous la première moulure amorçant le rhapiteau. Pieds des lettres à $40 \mathrm{~mm}$ environ au-dessus du bas du bloc; ce secteur est très meurtri,

21 P.-R. Gior, Les stèles armoricaines de l'Age du Fer dans Comptes rendus du XIV Congrès Préhistorique de France, Monaco, 1959 (Paris, 1965), p. 578-585, 3 fig. - Id., l.es civilisations de l'Age du Fer en Armorique dans La Préhistoire Française, II, C.X.R.S., 1976, p. 781-788.

22 Bouches-du-Rhòne : cinq à Glanum, Saint-Rémy-deProvence, cf. dans M. I.ejeune, R.I.G., I, G. $68 \mathrm{et} 70$ à 73 ; Vaucluse : trois à Cavaillon, ibid., G. 118 et 121, peut-être G. 119 el 120 .

23 D. Diнгх, Die Bauliunft der Giriechen, Leipzig, 1910, p. 127 , fig. 141 .
24 Vaucluse : Saint-Saturnin-d'Apt, cf. M. I.rJbune, R.I.G., I, G. $15 \%$.

25 Hérault: Montagnac, ibid., G. 224.

- Abréviatioss : $\mathrm{G}$ - = indicatif des lextes gallo-grecs de R.I.G. I - Ho. = A. Holder, Altceltischer Sprachschatz (Ieipzig, I, 1896 ; II, 1904 ; 11I, 1907 ; réimpression : Graz, 1961-1962). $-I E W=$ J. Pokorny, Indogermanisches etymologisches Wörterbuch, Bern, 1959. - KGP $=$ K. H. Schmidt, Die Komposition in gallischen Personennamen $=$ Zeitschrift für Celtische Philologie XXVI (1957), p. 33-301 ; cité ici avec la pagination de ZCPh. - R.I.G. = Recueil des inscriplions 


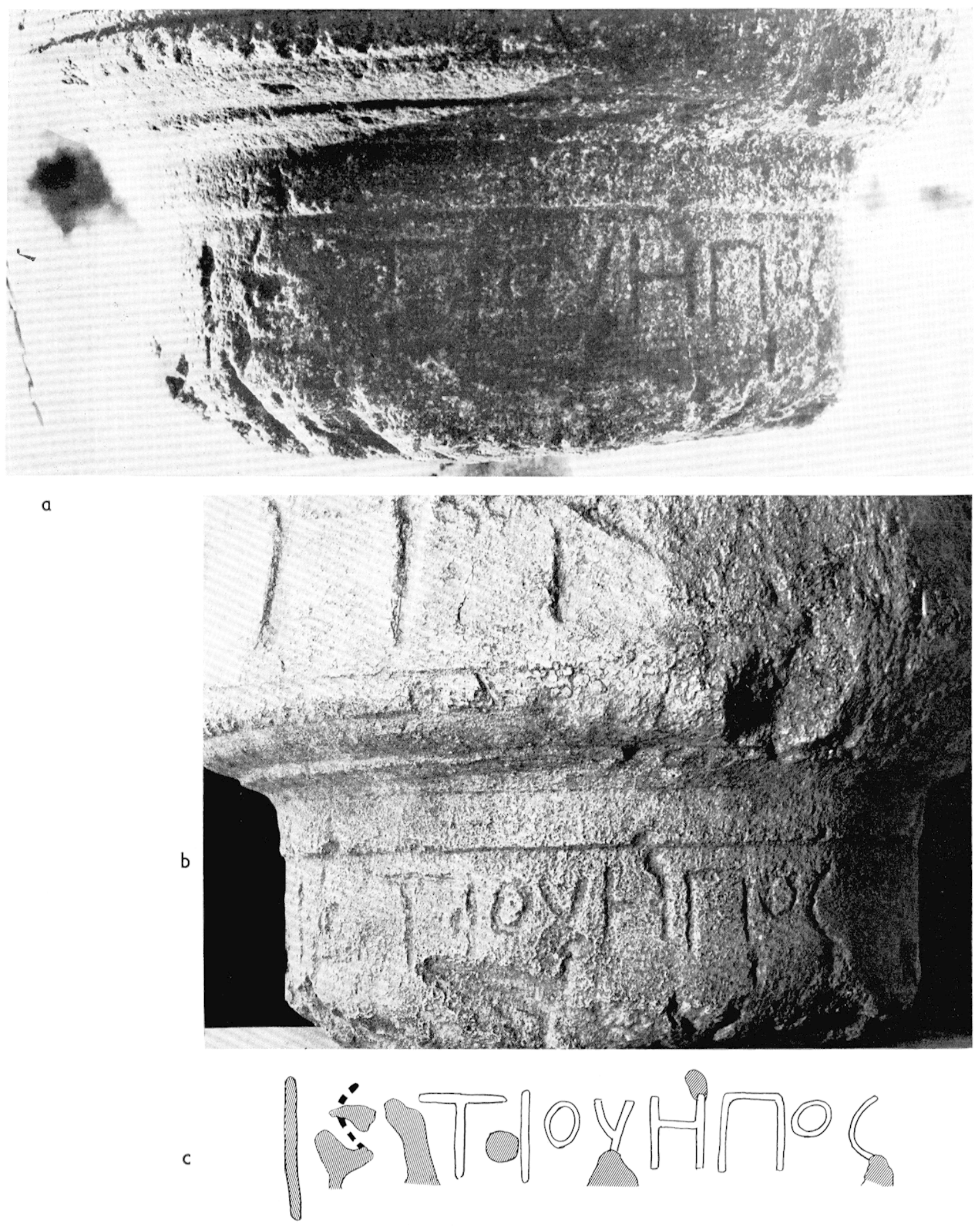

8 a : l'inscription; b : le moulage ; c : relevé graphique de l'inscription. 
mais aucune trace de lettre n'y apparait : au surplus, on imagine mal que le lapicide eût gravé une fraction de texte à cheval sur la jointure de deux tambours. On tiendra donc pour quasi certain que l'inscription tenait dans la seule ligne, très courte, qui nous est conservée.

Elle se lit :

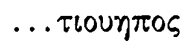

Entre $\tau$ et $\iota$, une tache végétale circulaire avait donné aux premiers lecteurs du texte l'impression

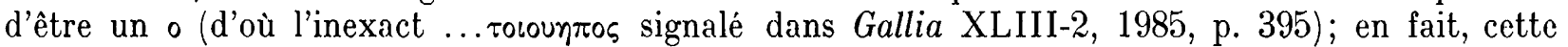
apparence ne correspond à aucune gravure.

Si la fin du texte est nette (blanc suivant ...os), son début ne l'est pas, et l'on peut se demander si l'inscription commence ou non avec $\tau \iota$, car la pierre a été là particulièrement malmenée par le chocs répétés d'instruments aratoires. Si la très grande sabrure verticale à gauche du haut du $\tau$ ne peut pas être un $\iota$, en revanche pourrait être un $\varepsilon$ lunaire (à barrette oblitérée par une meurtrissure) le creux semi-circulaire visible à gauche du haut du $\tau$ (encore que cet $\varepsilon$ serait imparfaitement aligné sur les lettres suivantes). On ne peut trancher. Retenir comme également possible une lecture

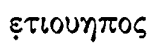

Le texte, se réduisant à un nom d'homme au nominatif, définit par là le monument comme funéraire et non votif. Le nom du défunt appelle plusieurs observations.

a) L'écriture, avec $\varepsilon, \sigma$ lunaires, assigne le document au gallo-grec "récent " (ce qui demeure très imprécis; en gros, $-150 /-50$ ); c'est à l'analyse stylistique du chapiteau qu'on devra demander, s'il se peut, une datation moins vague. - Sur l'emploi (non constant dans un même textc, voire dans

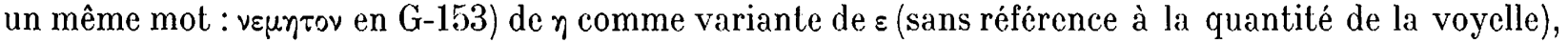
voir $R I G \mathrm{I}, \mathrm{p} .442 \mathrm{sv}$.

b) Le nom Etivepos est nouveau. C'est manifestement un composé.

c) Sur l'élément vepo-, voir $K G P$ 290. Il n'était jusqu'ici connu en composition que comme

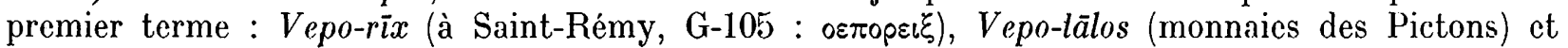
autres exemples hors de Gaule (Ho. III 177 sv.). Le voici attesté donc désormais aussi bien comme second terme.

d) Le premier terme fait difficulté. Une lecture $T i$-ne mène à rien. La lecture $E t i$ - permet au moins de formuler une hypothèse : il s'agirait du préverbe *eti- (skr. ali-, phryg. $\varepsilon \tau-$, got. id-, v. pr. el-, v. breton $e t-$-). Si on ne l'a pas encore rencontré dans les noms propres composés du vieux celtique, du moins apparait-il (sous forme apocopéc) dans des noms illyriens comme Et-trilus, El-leva (personnages du $-\mathrm{II}^{\mathrm{e}}$ s. cités par Tite-Live XLIV 30.3, 32.3). Il est incertain, en revanche, qu'on doive le reconnaître dans les noms de personnages mycéniens e-ti-me-de (PY Fn 324.1) et e-ti-ra-wo (PY Cn 130.10, 655,9; Sa 1264), car les usages orthographiques du linéairc B laissent loisibles les deux

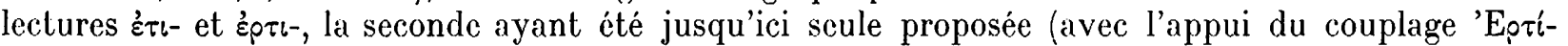
$\left.\lambda \bar{\alpha} F_{O \zeta} / \Lambda \bar{\alpha}(\xi)-\varepsilon \dot{\varepsilon} \tau \eta \bar{s}\right)$.

Si l'on admet cette analyse, on pourra se demander si le graffite Jovignot d'Alise-Sainte-Reinc

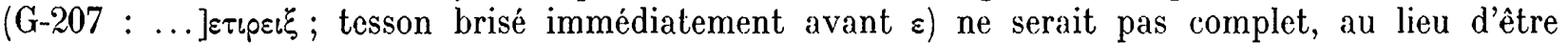

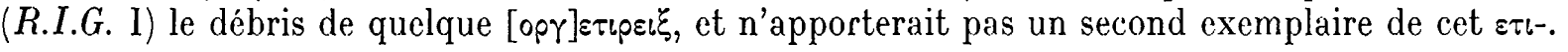

M. L.

gauloises (I, XLVc Supplément à Gallia, 1985, M. Lejeune, Textes gallo-grecs).
N.B. L'illustration photographique a été fournic par le Musée Municipal de Beaucaire. 